\title{
Antioxidant effects of hydroxysafflor yellow A and acetyl-11-keto- $\beta$-boswellic acid in combination on isoproterenol-induced myocardial injury in rats
}

\author{
MINCHUN CHEN $^{1,2^{*}}$, MINGMING WANG $^{1 *}$, QIONG YANG $^{1 *}$, MIN WANG $^{2}$, ZHIPENG WANG $^{2}$, YANRONG ZHU $^{1}$, \\ YIKAI ZHANG ${ }^{1}, \mathrm{CHAO} \mathrm{WANG}^{1}$, YANYAN JIA ${ }^{1}$, YUWEN LI $^{1}$ and AIDONG WEN ${ }^{1}$ \\ ${ }^{1}$ Department of Pharmacy, Xijing Hospital, ${ }^{2}$ Department of Pharmacology, College of Pharmacy, \\ The Fourth Military Medical University, Xi'an, Shaanxi 710032, P.R. China
}

Received May 5, 2015; Accepted April 5, 2016

DOI: $10.3892 /$ ijmm.2016.2571

\begin{abstract}
Oxidative stress plays an important role in the initiation and development of myocardial injury (MI). The peroxisome proliferator-activated receptor gamma coactivator-1 $\alpha$ (PGC-1 $\alpha)$ /nuclear factor erythroid 2-related factor 2 (Nrf2) pathway is considered to be a potential target for cardioprotection in MI. Acetyl-11-keto- $\beta$-boswellic acid (AKBA) is the major organic acid component extracted from Boswellia serrata Roxb. ex Colebr. Hydroxysafflor yellow A (HSYA) is the principal active constituent of Carthamus tinctorius L. In the present study, we aimed to investigate the cardioprotective effects of HSYA and AKBA in combination in vivo and in vitro, as well as the underlying mechanisms responsible for these effects. For this purpose, MI was produced in Sprague-Dawley rats by subcutaneous injection with isoproterenol. To model ischemic-like conditions in vitro, $\mathrm{H} 9 \mathrm{C} 2$ cells were subjected to oxygen-glucose deprivation (OGD). The levels of creatine kinase-MB (CK-MB), lactate dehydrogenase (LDH), malondial-
\end{abstract}

Correspondence to: $\mathrm{Dr}$ Yuwen Li or Professor Aidong Wen, Department of Pharmacy, Xijing Hospital, The Fourth Military Medical University, 127 Changlexi Street, Xi'an, Shaanxi 710032, P.R. China

E-mail: liyuwenzs@gmail.com

E-mail: adwen-2004@hotmail.com

*Contributed equally

Abbreviations: AKBA, acetyl-11-keto- $\beta$-boswellic acid; HSYA, hydroxysafflor yellow A; MI, myocardial injury; ROS, reactive oxygen species; SY, safflower yellow; ISO, isoproterenol; OGD, oxygenglucose deprivation; $\Delta \Psi \mathrm{m}$ or MMP, mitochondrial membrane potential; JC-1, 5,5',6,6'-tetraethylbenzimidazolylcarbocyanine iodide; PGC-1 $\alpha$, peroxisome proliferator-activated receptor gamma coactivator- $1 \alpha$; Nrf2, nuclear factor erythroid 2-related factor 2; CK-MB, creatine kinase-MB; LDH, lactate dehydrogenase; SOD, superoxide dismutase; MDA, malondialdehyde

Key words: acetyl-11-keto- $\beta$-boswellic acid, hydroxysafflor yellow A, myocardial injury, reactive oxygen species, oxidative stress dehyde (MDA) as well as superoxide dismutase (SOD) activity were examined as well as apoptotic cell death. Mitochondrial reactive oxygen species (ROS) production and mitochondrial membrane potential $\left(\Delta \Psi_{\mathrm{m}}\right.$ or MMP) were measured using MitoSOX Red and 5,5',6,6'-tetraethylbenzimidazolylcarbocyanine iodide (JC-1) dye. The expression of PGC-1 $\alpha$ and Nrf2 was quantified by western blot analysis and immunohistochemistry. HSYA and AKBA prevented myocardial pathological changes, significantly reduced the blood levels of CK-MB and LDH, and decreased apoptotic cell death. They significantly increased the expression of PGC-1 $\alpha$ and Nrf2, and the activity of the antioxidant enzyme SOD and also decreased the levels of MDA and ROS. Moreover, the reduction in MMP was partly prevented by HSYA and AKBA. Taken together, these findings elucidate the underlying mechanisms through which HSYA and AKBA protect against MI. Additionally, HSYA and AKBA appear to act synergistically in order to exert cardioprotective effects.

\section{Introduction}

Insufficient blood supply to the myocardium may result in myocardial ischemia, injury or infarction, or all three. Myocardial injury (MI) leads to hypoxia and ultimately to myocardial necrosis (1). Although thrombolysis is one of the effective treatments currently available, only limited numbers of patients are eligible for treatment. It is therefore necessary to develop alternative effective treatments. The increased production of reactive oxygen species (ROS) is a major contributor to the initiation and development of ischemic MI (2). Excessive ROS production causes a number of adverse effects, including DNA mutagenesis, cellular dysfunction and lipid peroxidation, in the myocardium following ischemic injury (3). Thus, antioxidants are considered to exert protective effects against MI. The peroxisome proliferator-activated receptor gamma coactivator- $1 \alpha$ (PGC-1 $\alpha$ ) acts as a powerful suppressor of ROS production, and improves cardiac energy metabolism and delays the progression of heart failure (4-6). PGC-1 $\alpha$ plays a critical role in mitochondrial biogenesis, and is a major regulator of the nuclear respiratory factors (7). Nuclear factor erythroid 2-related factor 2 (Nrf2) upregulates the transcription of cytoprotective genes, that reduce damage 
from oxidative stress (8). The PGC- $1 \alpha / \mathrm{Nrf} 2$ pathway is a promising therapeutic target for heart disease $(9,10)$.

According to the Compendium of Surgery (Wai Ke Da Cheng), the combination of Carthamus tinctorius L. and Boswellia serrata Roxb. ex Colebr. is widely used to protect against ischemic diseases. The principal active constituent of $C$. tinctorius is safflower yellow (SY) (11). Hydroxysafflor yellow A (HSYA), a water-soluble monomer of $\mathrm{SY}$, is responsible for the main beneficial effects of SY (12). C. tinctorius extract is reported to improve cardiac function following myocardial ischemic injury by exerting antioxidant effects $(13,14)$. Acetyl-11-keto- $\beta$-boswellic acid (AKBA) is the major organic acid component extracted from $B$. serrata $(15,16)$. It is a pentacyclic triterpene which possesses antioxidant properties (17). In our previous study, we have demonstrated that AKBA protects against cerebral ischemia/reperfusion (I/R) injury in rats by activating the Nrf2 pathway in order to enhance the antioxidant capacity of brain tissue (18). However, the additional biochemical mechanisms responsible for the beneficial effects of HSYA and AKBA in the treatment of MI remain unclear. In addition, to the best of our knowledge, the synergistic cardioprotective effects of HSYA and AKBA in combination have not been investigated to date.

In the present study, we applied in vivo and in vitro ischemic paradigms to analyze the protective effects of HSYA and AKBA, alone and in combination. We aimed to provide evidence to elucidate the mechanisms through which HSYA and AKBA protect against MI.

\section{Materials and methods}

Materials. Isoproterenol hydrochloride (ISO) was purchased from Sigma-Aldrich. (St. Louis, MO, USA). HSYA and AKBA were purchased from the National Institute for the Control of Pharmaceutical and Biological Products (Beijing, China). The chemical structures of HYSA and AKBA are shown in Fig. 1.

Animals. Six-week old male Sprague-Dawley rats $(250 \pm 20 \mathrm{~g})$ were purchased from the Animal Research Center of the Fourth Military Medical University (Xi'an, China). The animals were maintained in air-conditioned animal quarters at a temperature of $22 \pm 2^{\circ} \mathrm{C}$ under a $12 \mathrm{~h}$ light $/ 12 \mathrm{~h}$ dark cycle with unlimited access to food and water. All procedures were approved by the Ethics Committee for Animal Experimentation of the Fourth Military Medical University. The rats were randomized into five groups each consisting of six rats: i) sham group; ii) ISO + vehicle group; iii) ISO + HSYA group; iv) ISO + AKBA group; v) ISO + HSYA + AKBA group. A rat model of focal MI was established using the method of ISO-induced myocardial necrosis. Briefly, ISO (100 mg/kg) was dissolved in saline and injected subcutaneously into the rats at $24 \mathrm{~h}$ intervals for 2 days (19). ISO-induced MI was confirmed by the measurement of elevated activity levels of cardiac markers, compared with those in the normal rats. AKBA and HSYA were first dissolved in $2 \mathrm{ml}$ of $0.5 \%$ dimethyl sulfoxide (DMSO) solvent, and then diluted with physiological saline. The rats in the ISO + HSYA group were administered HSYA (100 mg/kg) through intragastric tubes. The rats in the ISO + AKBA group were administered AKBA (100 mg/kg) through intragastric tubes. The rats in the ISO + HSYA + AKBA group were administered
HSYA $(50 \mathrm{mg} / \mathrm{kg})$ and AKBA $(50 \mathrm{mg} / \mathrm{kg})$ through intragastric tubes. The doses of HSYA and AKBA was selected based upon previous studies $(20,21)$. The rats in the sham and ISO + vehicle groups were administered orally $2 \mathrm{ml}$ of $0.5 \%$ DMSO through intragastric tubes. The rats were gavaged for 14 days, and then subcutaneously injected with ISO at $24 \mathrm{~h}$ intervals for 2 consecutive days on the 15 th and 16th day.

Cell culture. The rat $\mathrm{H} 9 \mathrm{C} 2$ cardiomyocyte cell line was obtained from the American Type Culture Collection (ATCC, Manassas, VA, USA) and cultured in Dulbecco's modified Eagle's medium (DMEM) supplemented with $10 \%$ (v/v) fetal bovine serum at $37^{\circ} \mathrm{C}$ in a $\mathrm{CO}_{2}$ incubator. The medium was replaced every 2 days, and the cells were subjected to experimental procedures at $80-90 \%$ confluence.

Oxygen-glucose deprivation (OGD) and reoxygenation in H9C2 cells. The H9C2 cells were randomly divided into five groups: i) sham group without any treatment; ii) OGD + vehicle group; iii) OGD + HSYA group $(10 \mu \mathrm{M})$; iv) OGD + AKBA group $(10 \mu \mathrm{M}) ; \mathrm{v})$ OGD + HSYA + AKBA group (HSYA, $5 \mu \mathrm{M}$ and AKBA, $5 \mu \mathrm{M})$. The H9C2 cells were pre-treated with the above-mentioned drugs for $24 \mathrm{~h}$. To simulate ischemic-like conditions in vitro, the cells were subjected to OGD for $4 \mathrm{~h}$. The OGD procedures were based on a previously described method (22). Briefly, the H9C2 cells were washed with a solution (5 mM HEPES, $137 \mathrm{mM} \mathrm{NaCl}, 4 \mathrm{mM} \mathrm{KCl,} 1 \mathrm{mM} \mathrm{MgCl}_{2}$, $\left.1.5 \mathrm{mM} \mathrm{CaCl}_{2}, \mathrm{pH} 7.0\right)$. The cells were incubated in glucose-free DMEM and then placed in a hypoxic incubator $\left(37^{\circ} \mathrm{C}, 95 \% \mathrm{~N}_{2}\right.$, $5 \% \mathrm{CO}_{2}$ ) (Billups-Rothenberg, Del Mar, CA, USA). After $4 \mathrm{~h}$ of anoxia, the cells were subjected to reoxygenation and cultured under normal conditions in a $\mathrm{CO}_{2}$ incubator for $20 \mathrm{~h}$.

Histopathology. At the end of the experimental period, the rats were anesthetized with hydral (400 mg/kg, i.p.). The hearts were rapidly excised. Following removal, the cardiac apex was immediately fixed in $4 \%$ paraformaldehyde, routinely processed and embedded in paraffin wax. The cardiac apex was stained with hematoxylin and eosin (H\&E) and examined under a light microscope (Olympus IX71; Olympus, Tokyo, Japan).

Measurement of MI markers in the serum. Following experimental treatment, artexrial blood was collected and centrifuged at $16,000 \mathrm{rpm}$ for $10 \mathrm{~min}$. The serum was used to assay creatine kinase-MB (CK-MB) and lactate dehydrogenase (LDH) activities. The levels of CK-MB and LDH were assayed using commercial kits purchased from Jiancheng Bioengineering Institute (Nanjing, China) according to the manufacturer's instructions. An enzyme-linked immunosorbent assay was used to detect CK-MB and LDH levels with a microplate reader at 450 and $490 \mathrm{~nm}$ (Thermo Fisher Scientific, Waltham, MA, USA). CK-MB and LDH levels were also measured in the H9C2 cells. We determined the levels of CK-MB and LDH in the culture medium.

TUNEL assay and Hoechst 33258 staining for detection of cell apoptosis. Cell apoptosis was analyzed by performing the TUNEL assay in vivo and Hoechst 33258 staining in vitro. The TUNEL assay was performed using an In Situ Cell Death Detection kit (KeyGen Biotech, Nanjing, China). The 


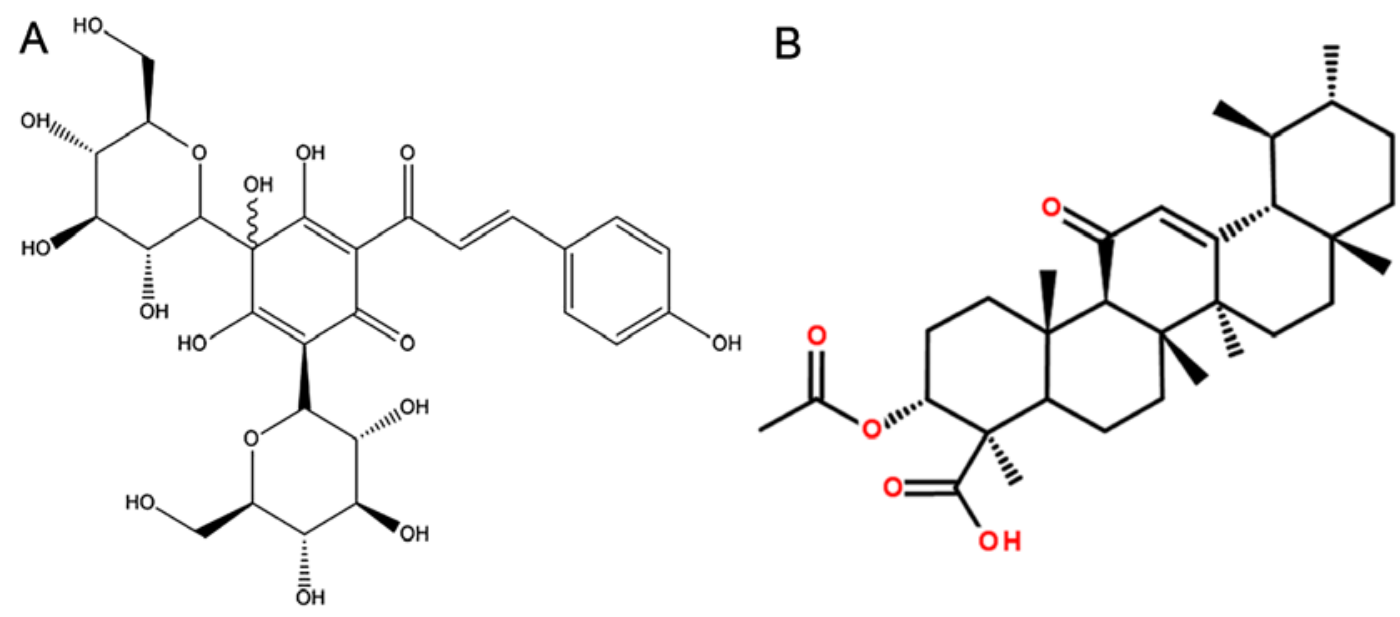

Figure 1. Chemical structure of (A) hydroxysafflor yellow A (HSYA; molecular weight, 612] and (B) acetyl-11-keto- $\beta$-boswellic acid (AKBA; molecular weight, 512).

paraffin-embedded tissue was washed twice with xylene for $10 \mathrm{~min}$, and then with distilled water and graded concentrations of ethanol (absolute, 95, 90, 80, 70 and 50\%). After washing with phosphate-buffered saline (PBS), the slides were immersed in a solution with $20 \mu \mathrm{g} / \mathrm{ml}$ proteinase $\mathrm{K}$ for $10 \mathrm{~min}$ at $37^{\circ} \mathrm{C}$, and then permeabilized in a solution with $0.1 \%$ Triton $\mathrm{X}-100$ and $0.1 \%$ sodium citrate for $5 \mathrm{~min}$. The cells were washed once with DAPI-methanol $(1 \mu \mathrm{g} / \mathrm{ml})$ and were then covered with DAPI-methanol and incubated for $15 \mathrm{~min}$ at $37^{\circ} \mathrm{C}$. After fluorescein staining with terminal deoxynucleotidyl transferase (TdT) and avidin-FITC, the individual nuclei were examined under a fluorescence microscope. The number of apoptotic nuclei was determined as a percentage of the total number of cells. An average of 300-400 nuclei were analyzed from each slide.

The H9C2 cells were stained with Hoechst 33258 and the nuclei were imaged. The AKBA/HSYA-treated H9C2 cells were washed twice with PBS, and incubated with $10 \mu \mathrm{M}$ Hoechst 33258 at room temperature for $30 \mathrm{~min}$. After three washes, the cells were grown on glass coverslips. The slides were examined for any nuclear morphological alterations and apoptotic bodies under an inverted fluorescence microscope (Olympus IX71; Olympus).

Detection of ROS. Mitochondrial ROS production in the $\mathrm{H} 9 \mathrm{C} 2$ cells was assessed using MitoSOX Red mitochondrial superoxide indicator (Invitrogen, Carlsbad, CA, USA). The H9C2 cells were seeded in a 6-well plate and grown to a density of $1 \times 10^{6} \mathrm{cell} / \mathrm{ml}$. The cells were loaded with $5 \mu \mathrm{M}$ MitoSOX Red for $15 \mathrm{~min}$ at $37^{\circ} \mathrm{C}$ in a $\mathrm{CO}_{2}$ incubator after $24 \mathrm{~h}$ of incubation with HSYA and $/$ or AKBA. The cells were then carefully washed twice with PBS. Fluorescence was read at $510 \mathrm{~nm}$ (excitation) and $580 \mathrm{~nm}$ (emission). The fluorescence intensity was quantified using ImageJ software (National Institutes of Health, Bethesda, MD, USA).

Assessment of mitochondrial membrane potential $\left(\Delta \Psi_{m}\right.$ or MMP). $\triangle \Psi \mathrm{m}$ was assessed using a flow cytometer (FACScan; Becton-Dickinson, Frankin Lakes, NJ, USA) using 5,5',6,6'-tetraethylbenzimidazolylcarbocyanine iodide (JC-1) dye (KeyGen Biotech) according to the manufacturer's instructions. The H9C2 cells were stained with JC-1 for $15 \mathrm{~min}$ at $37^{\circ} \mathrm{C}$ in a $\mathrm{CO}_{2}$ incubator following $24 \mathrm{~h}$ of incubation with
HSYA and/or AKBA. Green fluorescence was analyzed in the FL-1 (FITC) channel and red fluorescence was analyzed in the FL-2 (PE-A) channel. The fluorescence intensity was acquired for 10,000 events. The ratio of aggregated JC-1 and monomeric JC-1 represented the $\Delta \Psi \mathrm{m}$ of the H9C2 cells.

Evaluation of lipid peroxidation and antioxidant enzyme levels. After experimental treatment, the homogenates were centrifuged at $16,000 \mathrm{rpm}$ for 10 minutes. The resulting supernatant and the culture medium of the H9C2 cells was used to assay malondialdehyde (MDA) levels and superoxide dismutase (SOD) activity, according to the manufacturer's instructions, on a microplate reader at 560 and $532 \mathrm{~nm}$. The commercially available assay kits were purchased from Jiancheng Bioengineering Institute.

Immunohistochemical analysis. The paraffin sections $(5 \mathrm{~mm}$ thickness) were deparaffinaged in xylene, and then rehydrated with various grades of ethanol $(100,95,90,80$ and $70 \%)$. The sections were exposed to $3 \% \mathrm{H}_{2} \mathrm{O}_{2}$ for $10 \mathrm{~min}$ at $37^{\circ} \mathrm{C}$, and treated with citrate-buffered saline for $15 \mathrm{~min}$ at $95^{\circ} \mathrm{C}$. By incubating the sections in $10 \%$ bovine serum albumin, non-specific binding of immunoglobulins was blocked for $10 \mathrm{~min}$. Subsequently, the sections were incubated overnight at $4^{\circ} \mathrm{C}$ with primary antibodies, Nrf2 (ab31163, 1:1,000 dilution) or PGC-1 $\alpha$ (ab54481, 1:1,000 dilution) (both from Abcam, Cambridge, MA, USA) and then rinsed with PBS and incubated for $1 \mathrm{~h}$ with peroxidase-conjugated secondary antibody. The sections were visualized with 4',6-diamidino-2-phenylindole (DAPI) for nuclear counterstaining. Finally, the stained sections were examined under a fluorescence microscope (Olympus).

Protein extraction and western blot analysis. The cultured H9C2 cells were washed three times with ice-cold PBS and then harvested by scraping and centrifugation $(16,000 \mathrm{x} \mathrm{g}$ for $15 \mathrm{~min}$ at $4^{\circ} \mathrm{C}$ ). The sediment was lysed in Animal Cell Lysis solution containing $1 \mathrm{mM}$ protease inhibitor cocktail and phosphatase inhibitor mix (Tiandz, Inc., Beijing, China) on ice for $30 \mathrm{~min}$. The lysates were collected by centrifugation at $4^{\circ} \mathrm{C}$ for $15 \mathrm{~min}$ at $12,000 \mathrm{rpm}$. After quantifying the protein concentration using a Coomassie (Bradford) Protein Assay kit (Tiangen Biotech, Beijing, China) with a microplate reader at $595 \mathrm{~nm}$ 

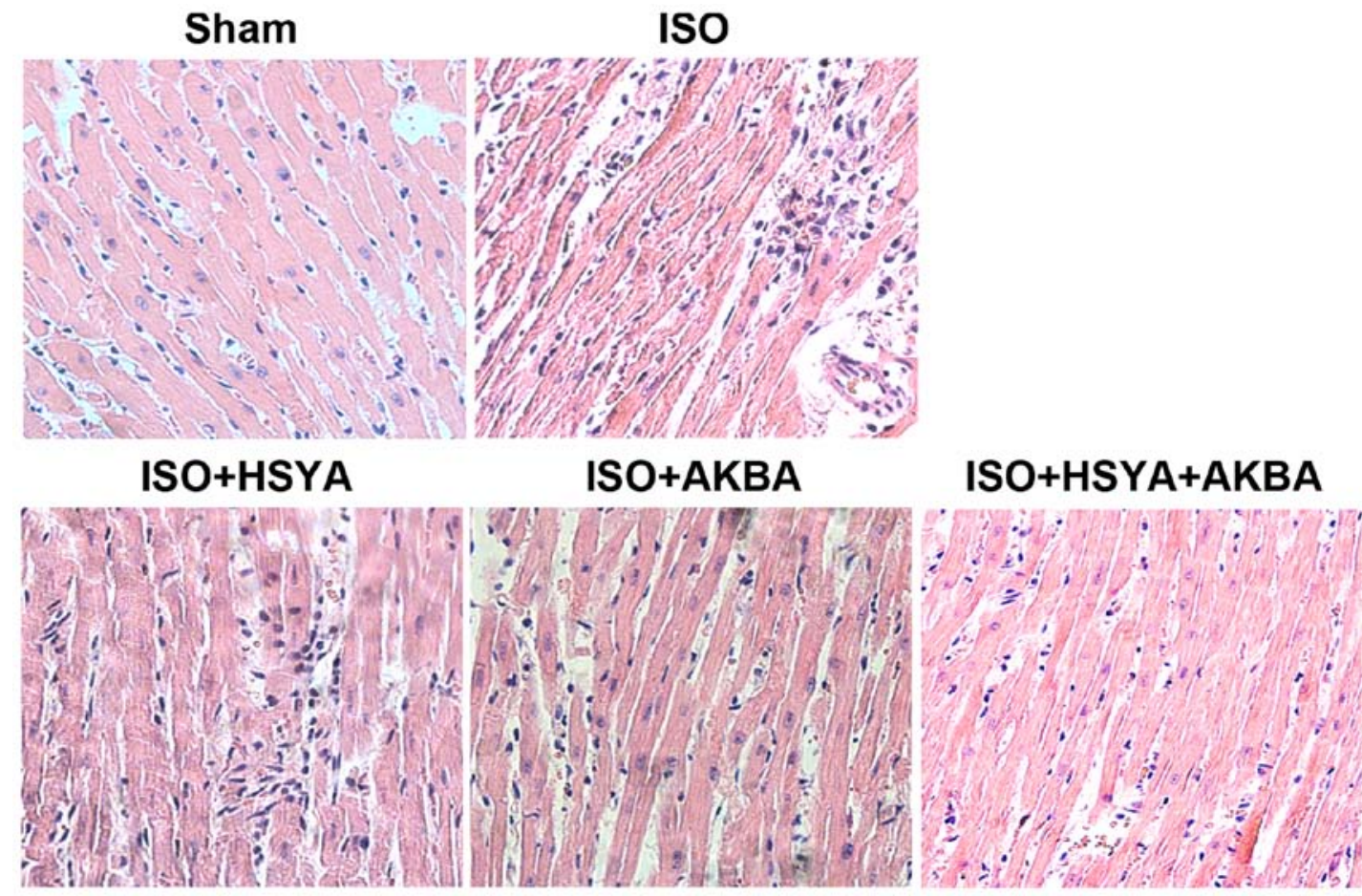

Figure 2. Hydroxysafflor yellow A (HSYA) and acetyl-11-keto- $\beta$-boswellic acid (AKBA) prevent isoproterenol hydrochloride (ISO)-induced pathological changes in rat heart tissue (heart tissues were stained with $\mathrm{H} \& \mathrm{E}$ and visualized under a light microscope at $\mathrm{x} 400$ magnification).

(Thermo Fisher Scientific), the lysates were boiled for $5 \mathrm{~min}$ at $100^{\circ} \mathrm{C}$ in loading buffer. Total protein $(20 \mu \mathrm{g})$ was subjected to $8 \%$ SDS-PAGE and transferred onto nitrocellulose membranes (Millipore Corp., Billerica, MA, USA), and blocked for $30 \mathrm{~min}$ at $37^{\circ} \mathrm{C}$ with $5 \%$ skim milk. The membranes were incubated with the respective primary antibodies, namely $\operatorname{Nrf} 2(1: 1,000$ dilution) and PGC-1 $\alpha$ (1:1,000 dilution) (both from Abcam) and $\beta$-actin (1:5,000 dilution; Sigma-Aldrich) overnight at $4^{\circ} \mathrm{C}$. After extensive washing, the membranes were incubated with horseradish peroxidase-conjugated secondary antibodies in TBST solution for $30 \mathrm{~min}$ at $37^{\circ} \mathrm{C}$, and then washed as described above. The bands were visualized with an ECL-Plus chemiluminescence kit (Millipore Corp.) and the signals were scanned and quantified by densitometric analysis (Bio-Rad, Richmond, CA, USA). $\beta$-actin was used as a total protein loading control.

Statistical analysis. Statistical analysis was performed by one-way analysis of variance (ANOVA) followed by a least significant difference (LSD) test for multiple comparisons, using SPSS version 13.0 statistical software. $\mathrm{P}<0.05$ was considered to indicate a statistically significant difference. All the results in this study are presented as the means \pm SD.

\section{Results}

HSYA and AKBA exert protective effects against MI. Histopathological analysis of the rat cardiac tissue was performed under a light microscope. Normal cardiac muscle fibers without necrosis, a branched appearance and continuity with adjacent myofibrils were observed in the sham group. Marked myofibrillar degeneration, necrosis, edema and infiltration with neutrophil granulocytes were found in the ISO-exposed group. However, HSYA and AKBA exerted protective effects against MI, and the combination of HSYA and AKBA appeared to be more effective (Fig. 2). ISO-induced MI caused the release of CK-MB and $\mathrm{LDH}$ into the bloodstream. Significant increases in the levels of CK-MB and LDH were detected in the ISO-exposed group compared with those in the sham group. Treatment with HSYA or AKBA reduced the ISO-mediated increase in the levels of CK-MB and LDH (Fig. 3A and B). To model ischemic-like conditions in vitro, H9C2 cells were subjected to transient OGD. HSYA and AKBA also markedly decreased CK-MB and LDH levels in the H9C2 cells following OGD (Fig. 3C and D). A combination of HSYA and AKBA exerted synergistic effects-in vivo and in vitro (Fig. 3).

Furthermore, the protective effects of HSYA and AKBA against myocardial damage were confirmed by TUNEL staining on sections of the rat myocardium isolated $48 \mathrm{~h}$ after the induction of ischemia by ISO (Fig. 4A). In the ISO-exposed group, TUNEL-positive cells were densely distributed in the myocardium. The HSYA- and AKBA-treated groups exhibited fewer numbers of TUNEL-positive cells. The percentage of TUNEL-positive cells in the ischemic myocardium decreased from 34.57 to $19.47 \%$ or $19.61 \%$ following treatment with HSYA or AKBA, respectively ( $\mathrm{n}=6$ rats/group; $\mathrm{P}<0.05$ ) (Fig. 4B). HSYA and AKBA in combination further decreased the percentage of TUNEL-positive cells compared with that in the HSYA or AKBA groups $(\mathrm{P}<0.05)$ (Fig. 4B). Representative photomicrographs of Hoechst 33258 staining are shown in Fig. 4C. The majority of the H9C2 cells in the group subjected to OGD appeared shrunken with triangulated, pycnotic nuclei. By contrast, the extent of cell damage was substantially reduced in the HSYA- and AKBA-treated groups (Fig. 4C).

HSYA and AKBA attenuate oxidative stress. Assessment of the mitochondrial ROS levels demonstrated that HSYA 

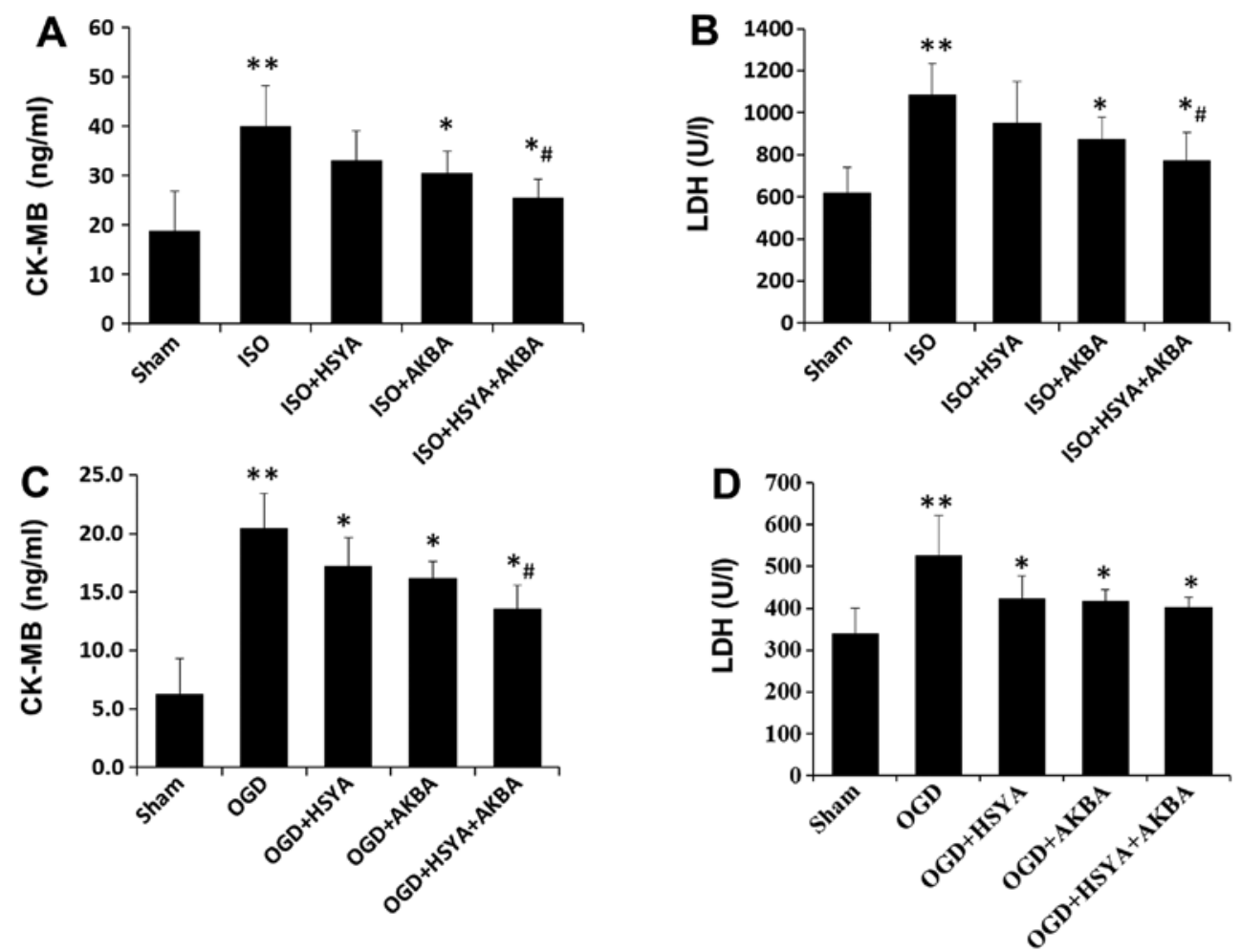

Figure 3. Hydroxysafflor yellow A (HSYA) and acetyl-11-keto- $\beta$-boswellic acid (AKBA) protect against myocardial injury (MI). The induction of MI by isoproterenol hydrochloride (ISO; $100 \mathrm{mg} / \mathrm{kg}$ ) was performed after treating the rats with either HSYA (100 mg/kg) or AKBA (100 mg/kg) alone, or a combination of HSYA $(50 \mathrm{mg} / \mathrm{kg}$ ) and AKBA $(50 \mathrm{mg} / \mathrm{kg})$ for 14 days. We measured (A) serum creatine kinase-MB (CK-MB) levels and (B) serum lactate dehydrogenase (LDH) levels. (C and D) HSYA and/or AKBA protected $\mathrm{H} 9 \mathrm{C} 2$ cells against oxygen-glucose deprivation (OGD)-induced cell damage. The H9C2 cells were subjected to OGD following treatment with either HSYA $(10 \mu \mathrm{M})$ or AKBA $(10 \mu \mathrm{M})$, or a combination of HSYA $(5 \mu \mathrm{M})$ and AKBA $(5 \mu \mathrm{M})$ for $24 \mathrm{~h}$. We measured (C) CK-MB activity and (D) LDH activity in the culture supernatant. The results are presented as the means \pm SD; "P<0.05 vs. ISO or OGD group; ${ }^{*} \mathrm{P}<0.05$ vs. HSYA or AKBA group; ${ }^{* *} \mathrm{P}<0.01$ vs. Sham group.

and AKBA effectively reduced the OGD-induced increase in mitochondrial ROS levels (Fig. 5A). The OGD-induced decrease in $\Delta \Psi \mathrm{m}$ was partly prevented by HSYA or AKBA (Fig. 5B). A combination of HSYA and AKBA induced a further decrease in ROS levels and an increase in $\Delta \Psi \mathrm{m}$ compared with the HSYA- or AKBA-treated H9C2 cells subjected to OGD (Fig. 5A and B). The MDA level, which is an index of lipid peroxidation, was increased in the groups exposed to ISO or OGD conditions compared with that in the sham groups. A reduction in the MDA level was observed in the HSYA- or AKBA-treated groups ( $\mathrm{n}=6$ rats/ group; $\mathrm{P}<0.05$ ) (Fig. 5C and $\mathrm{E}$ ). SOD activity was decreased in the groups exposed to ISO or OGD conditions compared with that in the sham groups, and was restored by HSYA or AKBA $(\mathrm{n}=6$ rats/group; $\mathrm{P}<0.05)$ (Fig. 5D and F). A combination of HSYA and AKBA induced a further decrease in the MDA level and a further increase in SOD activity in the groups which were exposed to ISO or OGD conditions (Fig. 5C-F).

HSYA and AKBA increase the expression of PGC-1 $\alpha$ and Nrf 2 . To determine whether PGC- $1 \alpha / \mathrm{Nrf} 2$ signaling is involved in the cardioprotective effects exerted by HSYA or AKBA, the expression of PGC- $1 \alpha$ and $\mathrm{Nrf} 2$ in the rat myocardium and the $\mathrm{H} 9 \mathrm{C} 2$ cells was evaluated by immunohistochemistry and western blot analysis, respectively. Immuhistochemical analysis of the myocardium revealed that HSYA or AKBA increased the expression of PGC-1 $\alpha$ and Nrf2 following ISO exposure (Fig. 6A and B). This was consistent with the results of western blot analysis which revealed that the expression of PGC- $1 \alpha$ and Nrf 2 in the $\mathrm{H} 9 \mathrm{C} 2$ cells was upregulated by HSYA or AKBA following exposure to OGD conditions (Fig. 6C). HSYA and AKBA in combination further enhanced the expression of PGC-1 $\alpha$ and $\mathrm{Nrf} 2$ in the $\mathrm{H} 9 \mathrm{C} 2$ cells compared with either HSYA or AKBA alone ( $\mathrm{n}=6$ rats/group; $\mathrm{P}<0.05$ ) (Fig. 6C).

\section{Discussion}

In the present study, we confirmed the protective effects of HSYA and AKBA against MI. Pre-treatment with HSYA and AKBA significantly inhibited tissue damage and cell death by decreasing mitochondrial ROS levels due to the increased expression of PGC- $1 \alpha$ and Nrf2. In addition, HSYA and AKBA in combination appeared to exert a synergistic cardioprotective effect.

Oxidative stress plays a critical role in the pathophysiology of myocardial ischemic injury (23). HSYA and AKBA are capable of neutralizing the increased mitochondrial ROS production (Fig. 5A). The first oxygen reduction product generated in mitochondria is superoxide $\left(\mathrm{O}_{2}{ }^{-}\right)$, which may be converted to $\mathrm{H}_{2} \mathrm{O}_{2}$ (24). To determine whether intracellular $\mathrm{O}_{2}^{-}$levels were decreased by HSYA and AKBA following MI, we stained the cells with MitoSOX Red, a superoxide-sensitive mitochondria-targeted hydroethidine analog. Taken together, these findings demonstrated that HSYA and AKBA enhanced 
A
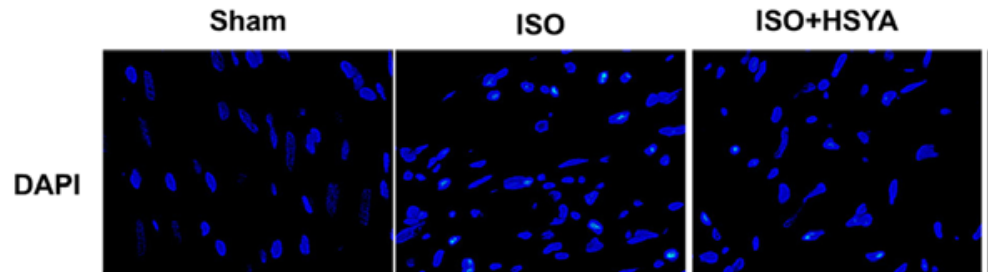

ISO+AKBA

ISO+HSYA+AKBA
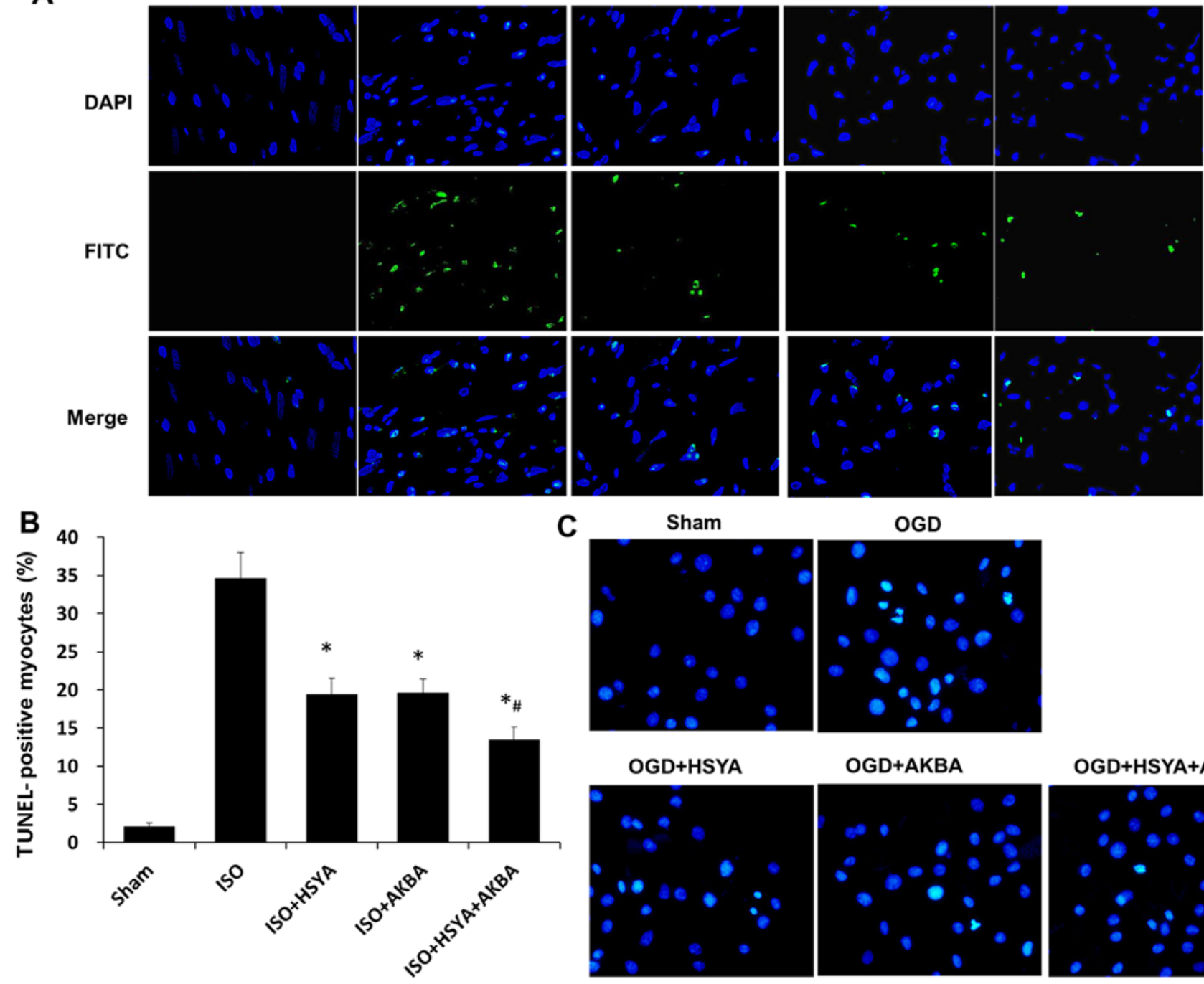

OGD+AKBA

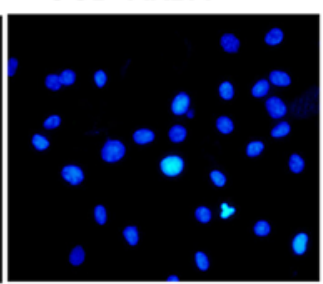

OGD+HSYA+AKBA

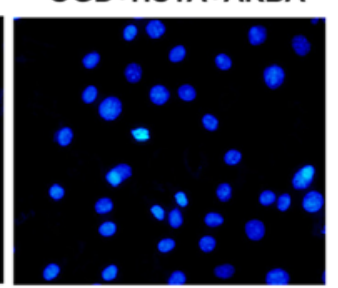

Figure 4. Pre-treatment with hydroxysafflor yellow A (HSYA) and acetyl-11-keto- $\beta$-boswellic acid (AKBA) inhibits apoptosis. (A) The induction of myocardial injury by isoproterenol hydrochloride (ISO; $100 \mathrm{mg} / \mathrm{kg}$ ) was performed after treating the rats with either HSYA (100 mg/kg) or AKBA (100 mg/kg) alone, or a combination of HSYA $(50 \mathrm{mg} / \mathrm{kg})$ and AKBA $(50 \mathrm{mg} / \mathrm{kg})$ for 14 days. The inhibitory effects of HSYA and AKBA on apoptosis in the rats exposed to ISO were evaluated using the TUNEL assay. (B) The quantitative results of TUNEL staining in rat hearts following different treatments are shown. (C) Effects of HSYA and AKBA on nuclear morphological changes in cardiomyocytes subjected to oxygen-glucose deprivation (OGD). The H9C2 cells were stained with Hoechst 33258 (magnification, $\mathrm{x} 400$ ). Results are presented as the means $\pm \mathrm{SD} ;{ }^{*} \mathrm{P}<0.05$ vs. ISO group; ${ }^{*} \mathrm{P}<0.05$ vs. HSYA or AKBA group.

the ability of cardiac mitochondria to decompose $\mathrm{H}_{2} \mathrm{O}_{2}$. Mitochondria are the principal source of endogenous ROS in the majority of mammalian cell types. Mitochondrial alterations are closely associated with ROS generation (25). During ischemia, mitochondria generate large quantities of ROS. However, excessive ROS production also damages mitochondria $(26,27)$. The destruction of mitochondrial integrity is associated with disruption of the $\Delta \Psi \mathrm{m}$. It has also been demonstrated that the increased permeabilization of the mitochondrial membrane may lead to increased intracellular ROS generation (28). Mitochondria are a key therapeutic target in MI. The results demonstrated that HSYA and AKBA largely maintained the $\Delta \Psi \mathrm{m}$ following OGD (Fig. 5B).

The antioxidant system is a compensatory mechanism for hyperoxidation which protects against oxidative injury. The effective ROS scavenger, SOD, is therefore essential for protective tissue functions. SOD is one of the major $\mathrm{H}_{2} \mathrm{O}_{2-}$ inducible antioxidant enzymatic defenses, which plays an important role in neutralizing oxygen free radicals (29). MDA is not only generated by oxidative stress-induced peroxidation but also by the interaction between free radical or lipid peroxyl radicals ( $\mathrm{LOO}^{*}$ ) with lipid molecules (30). In the present study, the reduced activity of SOD and the increased levels of MDA were due to increased oxidative stress following ISO-induced MI. Reduced SOD activity and increased MDA levels may be attributable to the accumulation of free radicals in the heart and irreversible depletion of the endogenous antioxidant system. Consistent with previous findings (31), a significant increase in SOD activity and a decrease in MDA levels were observed in the HSYA or AKBA groups, in the present study (Fig. 5). HYSA and AKBA reduced ROS levels, enhanced SOD activity and inhibited lipid peroxidation, therefore alleviating MI.

Mitochondria are the principal sites of ROS production (32). PGC-1 $\alpha$ and Nrf2 are the major regulators of mitochondrial biogenesis and activity $(6,7)$. PGC-1 $\alpha$ and Nrf2 regulate the expression of a set of antioxidant-related genes which remove 

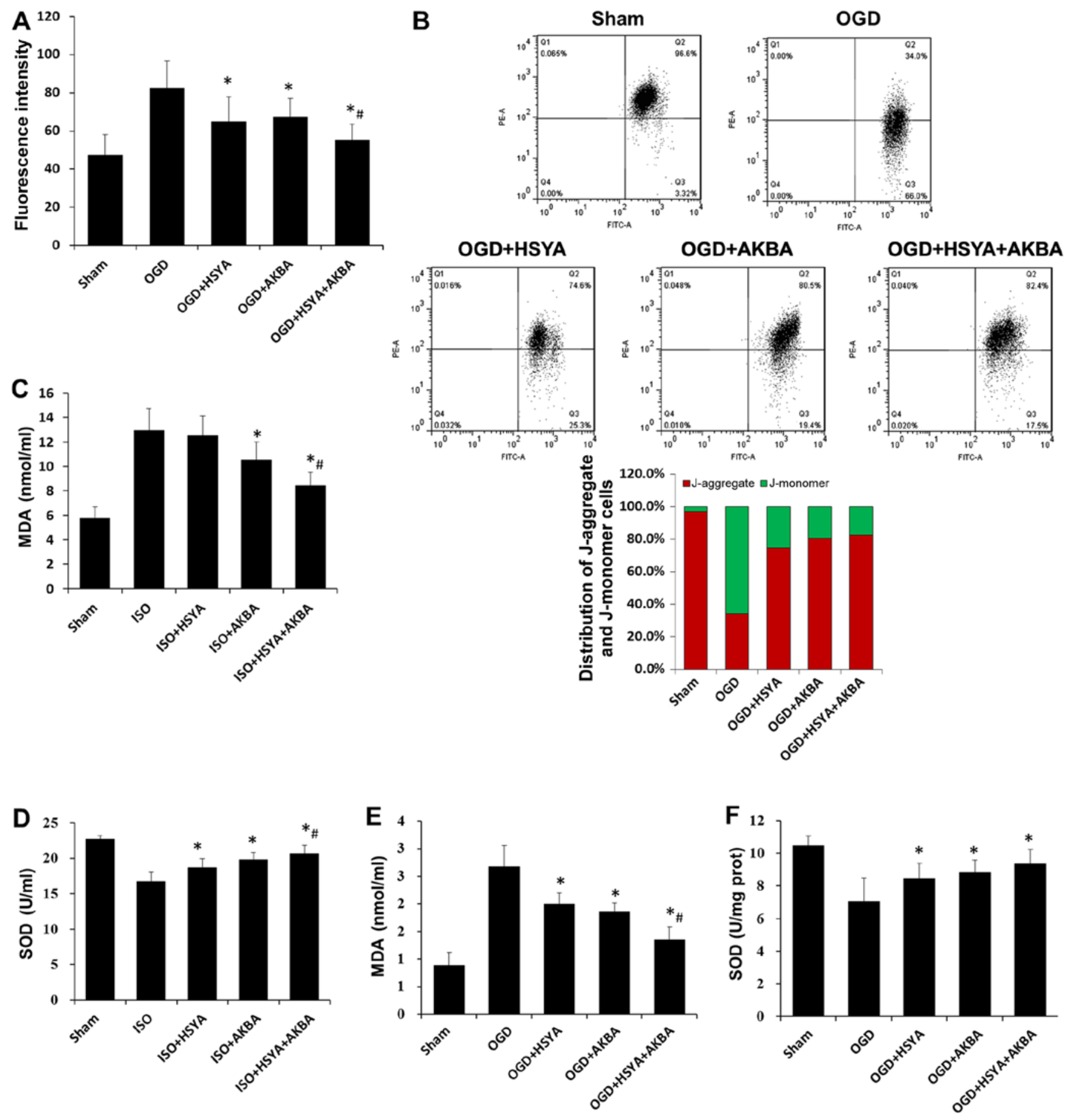

Figure 5. Hydroxysafflor yellow A (HSYA) and acetyl-11-keto- $\beta$-boswellic acid (AKBA) attenuate oxidative stress. H9C2 cells were treated with HSYA $(10 \mu \mathrm{M})$ and AKBA $(10 \mu \mathrm{M})$ for $24 \mathrm{~h}$ prior to oxygen-glucose deprivation (OGD) for $4 \mathrm{~h}$, followed by incubation under normal conditions. Data are presented as the means \pm SD of five independent experiments. (A) Intracellular reactive oxygen species (ROS) level. (B) The cells were incubated with JC-1 (10 ng/ml) for $30 \mathrm{~min}$, and mitochondrial membrane potential ( $\Delta \Psi \mathrm{m}$ or MMP) was analyzed by flow cytometry. The bar diagram shows the percentage of red fluorescence to green fluorescence. (C and D) The rats were treated with either HSYA $(100 \mathrm{mg} / \mathrm{kg})$ or AKBA $(100 \mathrm{mg} / \mathrm{kg})$ alone, or a combination of $\mathrm{HSYA}(50 \mathrm{mg} / \mathrm{kg})$ and AKBA $(50 \mathrm{mg} / \mathrm{kg}$ ) for 14 days prior to the administration of isoproterenol hydrochloride (ISO;100 mg/kg) for 2 days, to induce myocardial injury. Determination of (C) malondialdehyde (MDA) levels and (D) superoxide dismutase (SOD) activity in the rat myocardium. Determination of (E) MDA levels and (F) SOD activity under OGD conditions. ${ }^{*} \mathrm{P}<0.05$ vs. ISO or OGD group; ${ }^{\#} \mathrm{P}<0.05$ vs. HSYA or AKBA group.

ROS through sequential enzymatic reactions $(9,33)$. Thus, they are the key components involved in the maintenance of cellular redox homeostasis following oxidative stress-induced MI (10). Consequently, compounds that interfere with the PGC-1 $\alpha$ / Nrf2 pathway may have the potential to be used as cardioprotective agents. It has been previously demonstrated that HSYA exerts neuroprotective effects against cerebral I/R injury through its antioxidant action (34). HSYA has been reported to protect $\mathrm{H} 9 \mathrm{C} 2$ cardiomyocytes against apoptosis through the PI3K/Akt/Nrf2 pathway (14). Additionally, AKBA was demonstrated to exert antioxidant effects thereby protecting against cerebral ishemic injury in the rat brain through the 
A

Sham

ISO

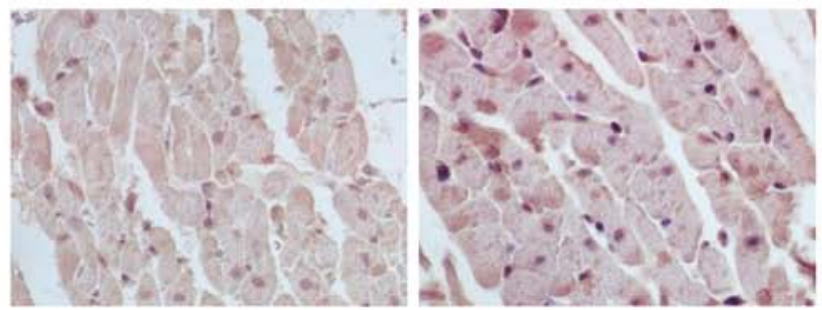

ISO+HSYA

ISO+AKBA

ISO+HSYA+AKBA
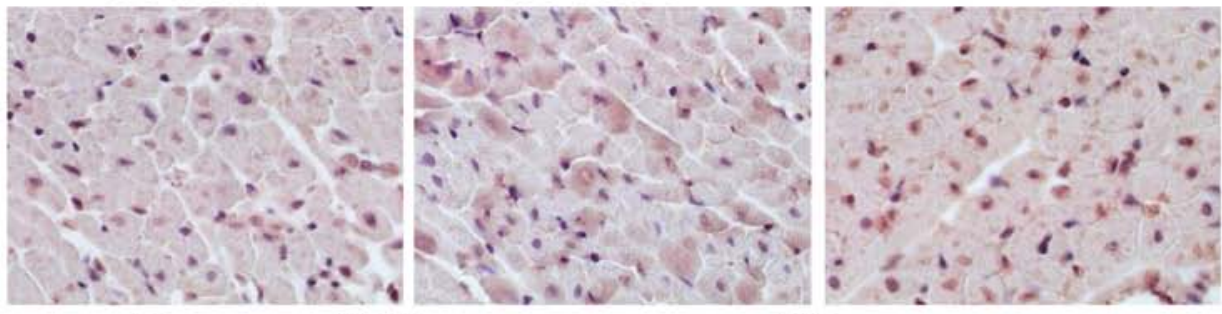

B

Sham

ISO

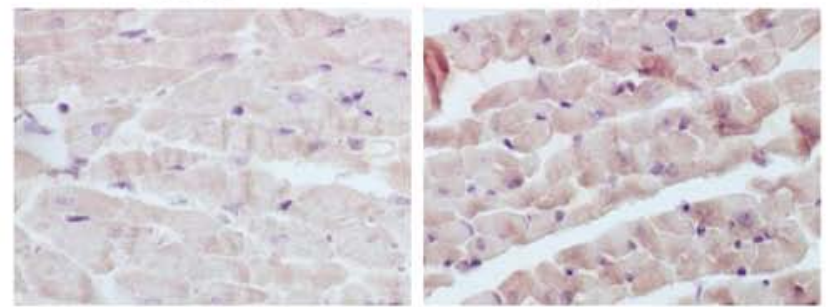

ISO+HSYA

ISO+AKBA

ISO+HSYA+AKBA

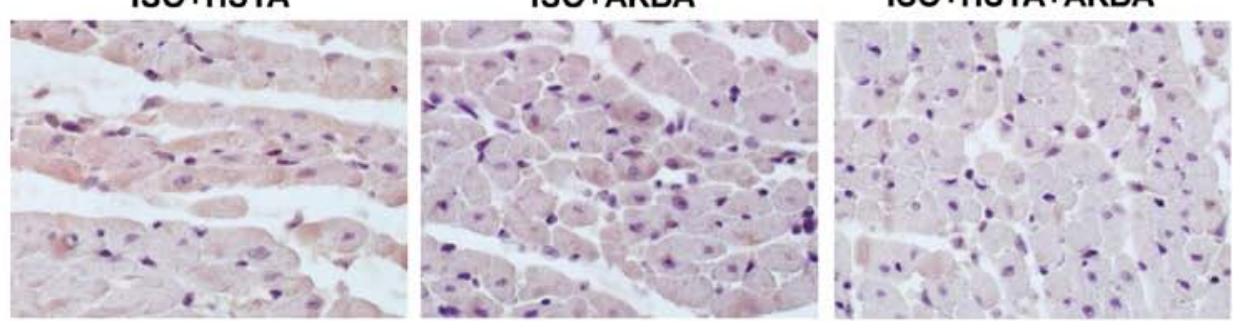

C
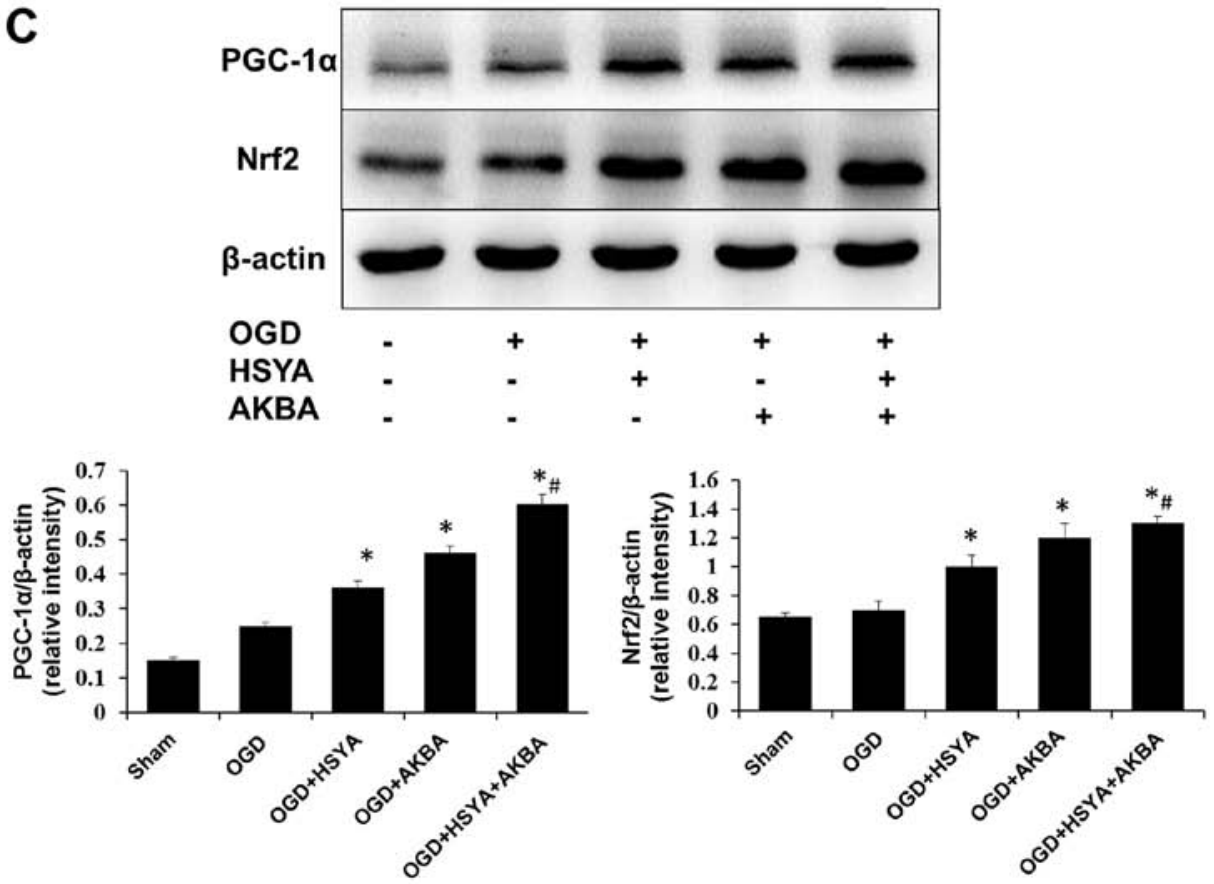

Figure 6. Expression of peroxisome proliferator activated receptor gamma coactivator-1 $\alpha$ (PGC-1 $\alpha$ ) and nuclear factor erythroid 2-related factor 2 (Nrf2). (A) PGC-1 $\alpha$ and (B) Nrf2 expression was induced by hydroxysafflor yellow A (HSYA) and/or acetyl-11-keto- $\beta$-boswellic acid (AKBA) in the rats with isoproterenol hydrochloride (ISO)-induced MI (magnification, x400). Immunohistochemical analysis was performed on the tissue samples in order to determine Nrf2 and PGC-1 $\alpha$ expression. (C) PGC-1 $\alpha$ and Nrf2 expression in cultured H9C2 cells was evaluated by western blot analysis. The intensity of each band was quantified by densitometry. Data represent the means $\pm \mathrm{SD} ;{ }^{*} \mathrm{P}<0.05$ compared with oxygen-glucose deprivation (OGD) group; ${ }^{\#} \mathrm{P}<0.05$ vs. HSYA or AKBA group. 
Nrf2/heme oxygenase-1 (HO-1) pathway (18). Consequently, it is important to examine the antioxidant activity of HSYA and AKBA and the role of these compounds in modulating the PGC-1 $\alpha /$ Nrf 2 pathway. In the present study, HSYA and AKBA markedly increased the expression of PGC-1 $\alpha$ and nuclear $\mathrm{Nrf} 2$ in rats with ISO-induced MI as well as in the $\mathrm{H} 9 \mathrm{C} 2$ cells subjected to OGD. The activation of PGC-1 $\alpha$ and Nrf2 is known to stimulate mitochondrial biogenesis and to activate enzymes. Following nuclear translocation, PGC-1 $\alpha$ promotes the expression of its target genes and thus, maintains the basal and inducible expression of a number of antioxidant genes. It has been found that PGC- $1 \alpha$ relies on docking to particular transcription factors in order to perform different biological programs (35). The transcriptional activation of mitochondrial biogenesis is dependent on docking of Nrf2. Increased Nrf2 activity is associated with an enhanced antioxidant defenses and tolerance to stress-induced tissue pathologies (36).

In clinical practice, the concept of multidrug interventions for MI has received much attention (37-39). Drugs act synergistically when lower concentrations of both drugs produce better efficacy than monotherapy with either agent. The synergistic mode of action of drugs should be given priority when multidrug therapy is indicated. In comparison with monotherapy, the potential advantages of the combination drug therapy are: i) lower doses of drugs improve treatment efficacy; ii) reduced adverse effects and drug toxicities. The present study demonstrated the synergistic actions of HSYA and AKBA to produce cardioprotective effects (Figs. 2-4). In support of this notion, the administration of HSYA and AKBA in combination exerted synergistic antioxidant effects (Fig. 5). Western blot analysis revealed the synergistic effects of AKBA and HSYA on PGC-1 $\alpha$ expression in vitro (Fig. 6C). HSYA and AKBA in combination decreased mitochondrial superoxide production and largely maintained $\Delta \Psi \mathrm{m}$, thus, leading to further protection against MI compared with HSYA or AKBA alone.

Overall, the results of the present study have demonstrated that HSYA and AKBA are effective in protecting against MI by alleviating mitochondria-dependent oxidative stress, through the enhanced expression of PGC-1 $\alpha$ and Nrf2. In addition, HSYA and AKBA appear to exert synergistic cardioprotective effects.

\section{Acknowledgements}

The present study was supported by grants from the National Natural Science Foundation of China (nos. 81373947 and 81201985 ) and the Key Technologies for New Drug Innovation and Development of China (nos. 2011ZXJ09202-13 and 2012BAK25B00).

\section{References}

1. Anversa P and Sonnenblick EH: Ischemic cardiomyopathy: pathophysiologic mechanisms. Prog Cardiovasc Dis 33: 49-70, 1990.

2. Eltzschig HK and Eckle T: Ischemia and reperfusion - from mechanism to translation. Nat Med 17: 1391-1401, 2011.

3. Takimoto E and Kass DA: Role of oxidative stress in cardiac hypertrophy and remodeling. Hypertension 49: 241-248, 2007.

4. Zhang J, Wei C, Wang H, Tang S, Jia Z, Wang L, Xu D and Wu Y: Protective effect of qiliqiangxin capsule on energy metabolism and myocardial mitochondria in pressure overload heart failure rats. Evid Based Complement Alternat Med 2013: 378298, 2013.
5. Wan Z, Root-McCaig J, Castellani L, Kemp BE, Steinberg GR and Wright DC: Evidence for the role of AMPK in regulating PGC-1alpha expression and mitochondrial proteins in mouse epididymal adipose tissue. Obesity (Silver Spring) 22: 730-738, 2014.

6. Lai L, Wang M, Martin OJ, Leone TC, Vega RB, Han X and Kelly DP: A role for peroxisome proliferator-activated receptor $\gamma$ coactivator 1 (PGC-1) in the regulation of cardiac mitochondrial phospholipid biosynthesis. J Biol Chem 289: 2250-2259, 2014.

7. Wu Z, Puigserver P, Andersson U, Zhang C, Adelmant G Mootha V, Troy A, Cinti S, Lowell B, Scarpulla RC and Spiegelman BM: Mechanisms controlling mitochondrial biogenesis and respiration through the thermogenic coactivator PGC-1. Cell 98: 115-124, 1999.

8. Cimino F, Speciale A, Anwar S, Canali R, Ricciardi E, Virgili F, Trombetta D and Saija A: Anthocyanins protect human endothelial cells from mild hyperoxia damage through modulation of Nrf2 pathway. Genes Nutr 8: 391-399, 2013.

9. Zhou S, Sun W, Zhang Z and Zheng Y: The role of Nrf2-mediated pathway in cardiac remodeling and heart failure. Oxid Med Cell Longev 2014: 260429, 2014.

10. Hybertson BM, Gao B, Bose SK and McCord JM: Oxidative stress in health and disease: the therapeutic potential of Nrf2 activation. Mol Aspects Med 32: 234-246, 2011.

11. Nie PH, Zhang L, Zhang WH, Rong WF and Zhi JM: The effects of hydroxysafflor yellow A on blood pressure and cardiac function. J Ethnopharmacol 139: 746-750, 2012.

12. Feng ZM, He J, Jiang JS, Chen Z, Yang YN and Zhang PC: NMR solution structure study of the representative component hydroxysafflor yellow A and other quinochalcone C-glycosides from Carthamus tinctorius. J Nat Prod 76: 270-274, 2013.

13. Han SY, Li HX, Ma X, Zhang K, Ma ZZ and Tu PF: Protective effects of purified safflower extract on myocardial ischemia in vivo and in vitro. Phytomedicine 16: 694-702, 2009.

14. Liu SX, Zhang Y, Wang YF, Li XC, Xiang MX, Bian C and Chen P: Upregulation of heme oxygenase-1 expression by hydroxysafflor yellow A conferring protection from anoxia/reoxygenation-induced apoptosis in $\mathrm{H} 9 \mathrm{c} 2$ cardiomyocytes. Int $\mathrm{J}$ Cardiol 160: 95-101, 2012.

15. Park B, Sung B, Yadav VR, Cho SG, Liu M and Aggarwal BB: Acetyl-11-keto- $\beta$-boswellic acid suppresses invasion of pancreatic cancer cells through the downregulation of CXCR4 chemokine receptor expression. Int J Cancer 129: 23-33, 2011.

16. Wang Y, Sun Y, Wang C, Huo X, Liu P, Wang C, Zhang B, Zhan L, Zhang H, Deng S, et al: Biotransformation of 11-keto- $\beta$-boswellic acid by Cunninghamella blakesleana. Phytochemistry 96: 330-336, 2013.

17. Henkel A, Kather N, Mönch B, Northoff H, Jauch J and Werz O: Boswellic acids from frankincense inhibit lipopolysaccharide functionality through direct molecular interference. Biochem Pharmacol 83: 115-121, 2012.

18. Ding Y, Chen M, Wang M, Wang M, Zhang T, Park J, Zhu Y, Guo C, Jia Y, Li Y and Wen A: Neuroprotection by acetyl-11keto- $\beta$-Boswellic acid, in ischemic brain injury involves the Nrf2/HO-1 defense pathway. Sci Rep 4: 7002, 2014.

19. Stanely Mainzen Prince P and Roy AJ: p-Coumaric acid attenuates apoptosis in isoproterenol-induced myocardial infarcted rats by inhibiting oxidative stress. Int J Cardiol 168: 3259-3266, 2013.

20. Tian Y, Yang ZF, Li Y, Qiao Y, Yang J, Jia YY and Wen AD: Pharmacokinetic comparisons of hydroxysafflower yellow A in normal and blood stasis syndrome rats. J Ethnopharmacol 129: $1-4,2010$.

21. Yadav VR, Prasad S, Sung B, Gelovani JG, Guha S, Krishnan S and Aggarwal BB: Boswellic acid inhibits growth and metastasis of human colorectal cancer in orthotopic mouse model by downregulating inflammatory, proliferative, invasive and angiogenic biomarkers. Int J Cancer 130: 2176-2184, 2012.

22. Woo AY, Cheng CH and Waye MM: Baicalein protects rat cardiomyocytes from hypoxia/reoxygenation damage via a prooxidant mechanism. Cardiovasc Res 65: 244-253, 2005.

23. Tomaselli GF and Barth AS: Sudden cardio arrest: oxidative stress irritates the heart. Nat Med 16: 648-649, 2010

24. Cadenas E and Davies KJ: Mitochondrial free radical generation, oxidative stress, and aging. Free Radic Biol Med 29: 222-230, 2000.

25. Green DR and Kroemer G: The pathophysiology of mitochondrial cell death. Science 305: 626-629, 2004.

26. Smith RA, Hartley RC, Cochemé HM and Murphy MP: Mitochondrial pharmacology. Trends Pharmacol Sci 33: 341-352, 2012. 
27. Chen Q and Lesnefsky EJ: Depletion of cardiolipin and cytochrome $c$ during ischemia increases hydrogen peroxide production from the electron transport chain. Free Radic Biol Med 40: 976-982, 2006.

28. Chen YR and Zweier JL: Cardiac mitochondria and reactive oxygen species generation. Circ Res 114: 524-537, 2014.

29. Warner DS, Sheng H and Batinić-Haberle I: Oxidants, antioxidants and the ischemic brain. J Exp Biol 207: 3221-3231, 2004.

30. Halliwell B and Gutteridge $J$ (eds): Free Radicals in Biology and Medicine. 1st edition. Oxford University Press, New York, NY, 2007.

31. Hartmann RM, Morgan Martins MI, Tieppo J, Fillmann HS and Marroni NP: Effect of Boswellia serrata on antioxidant status in an experimental model of colitis rats induced by acetic acid. Dig Dis Sci 57: 2038-2044, 2012.

32. Li J, Ma X, Yu W, Lou Z, Mu D, Wang Y, Shen B and Qi S: Reperfusion promotes mitochondrial dysfunction following focal cerebral ischemia in rats. PLoS One 7: e46498, 2012.

33. St-Pierre J, Drori S, Uldry M, Silvaggi JM, Rhee J, Jäger S, Handschin C, Zheng K, Lin J, Yang W, et al: Suppression of reactive oxygen species and neurodegeneration by the PGC-1 transcriptional coactivators. Cell 127: 397-408, 2006.
34. Wei X, Liu H, Sun X, Fu F, Zhang X, Wang J, An J and Ding H: Hydroxysafflor yellow A protects rat brains against ischemiareperfusion injury by antioxidant action. Neurosci Lett 386: 58-62, 2005.

35. Kressler D, Schreiber SN, Knutti D and Kralli A: The PGC-1related protein PERC is a selective coactivator of estrogen receptor alpha. J Biol Chem 277: 13918-13925, 2002.

36. Joshi G and Johnson JA: The Nrf2-ARE pathway: A valuable therapeutic target for the treatment of neurodegenerative diseases. Recent Patents CNS Drug Discov 7: 218-229, 2012.

37. Ma X, Oyamada S, Gao F, Wu T, Robich MP, Wu H, Wang X, Buchholz B, McCarthy S, Gu Z, et al: Paclitaxel/sirolimus combination coated drug-eluting stent: In vitro and in vivo drug release studies. J Pharm Biomed Anal 54: 807-811, 2011.

38. Gaziano TA, Galea G and Reddy KS: Scaling up interventions for chronic disease prevention: the evidence. Lancet 370: 1939-1946, 2007.

39. Robich MP, Chu LM, Oyamada S, Sodha NR and Sellke FW: Myocardial therapeutic angiogenesis: a review of the state of development and future obstacles. Expert Rev Cardiovasc Ther 9: 1469-1479, 2011. 